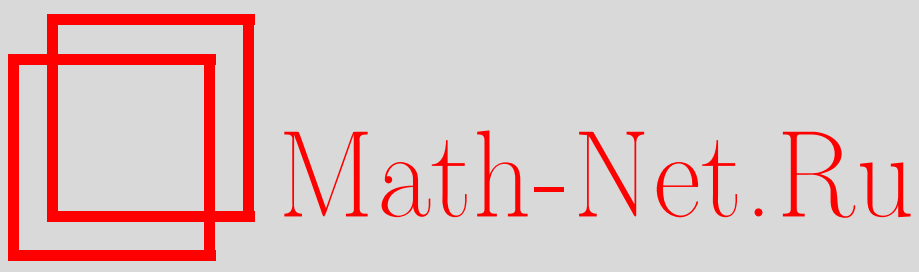

Ю. И. Кольцун, Волновые процессы при многоцикловом нагружении детали. Гипотеза плоских продольных сечений, Вестн. Сам. гос. техн. ун-та. Сер. Физ.-мат. науки, 2005, выпуск 34, 199-202

DOI: https://doi.org/10.14498/vsgtu358

Использование Общероссийского математического портала Math-Net.Ru подразумевает, что вы прочитали и согласны с пользовательским соглашением

http://www . mathnet.ru/rus/agreement

Параметры загрузки:

IP: 18.207 .199 .55

26 апреля 2023 г., 12:52:37

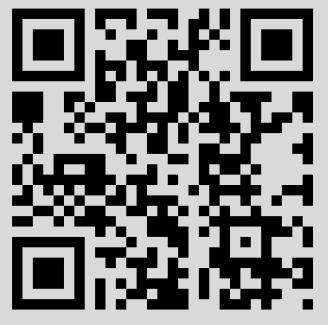




\title{
ВОЛНОВЫЕ ПРОЦЕССЫ ПРИ МНОГОЦИКЛОВОМ НАГРУЖЕНИИ ДЕТАЛИ. ГИПОТЕЗА ПЛОСКИХ ПРОДОЛЬНЫХ СЕЧЕНИЙ
}

\begin{abstract}
На реальных усталостных изломах показано существование упругих волн, возникающих при многоцикловом нагружении детали, и проявляющих своё существование в диапазоне от предела выносливости по первой макротрещине до предела выносливости по разрушению. Поверхность изломов свидетельствует о неэффективности гипотезы плоских поперечных сечений НавьеБернулли в указанном диапазоне многочиклового нагружения. С иелью создания расчетной методики построения (прогнозирования) поверхности усталостного излома предлагается гипотеза плоских продольных сечений, картина распределения которых в детали зависит от вида нагружения.
\end{abstract}

Прогнозирование поверхности усталостного излома детали на сегодняшний день не представляется возможным, с одной стороны, в силу основных гипотез и принципов механики сплошной среды, с другой стороны, в силу отсутствия результатов специальных экспериментальных исследований. На рис. 1 - 4 показаны усталостные изломы цилиндрических образцов из различных сплавов с элементами деталей в виде кольцевого надреза полукруглого профиля при симметричном изгибе с частотой 19,5 Гц. Видно, что при амплитуде напряжений $\sigma_{a}$, превышающей предел выносливости детали $\sigma_{-1 \partial}$ в полтора раза усталостный излом независимо от степени концентрации напряжений представляет собой плоское поперечное сечение. Это свидетельствует о том, что на данном уровне амплитуды выполняется гипотеза плоских поперечных сечений Навье-Бернулли [1,2 ], которая является основополагающим принципом механики сплошной среды.

Однако на практике при циклическом нагружении простыми видами деформаций-кручением, изгибом, центральным растяжением-сжатием, а также в условиях сложного нагружения при амп литудах напряжений до предела выносливости поверхность усталостных изломов представляет собой сложную картину, не поддающуюся предварительному математическому описанию, так как объективно гипотеза НавьеБернулли не выполняется. Возникает естественный вопрос: возможно ли математическое описание поверхностей усталостных изломов при различных видах деформаций ещё до разрушения деталей и какими принципами в этом случае руководствоваться?

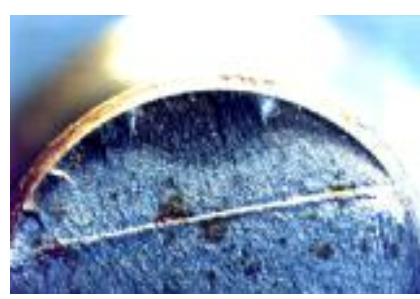

Р и с. 1. Сталь 45: $\sigma_{a}=1,5 \cdot \sigma_{-l \partial}$

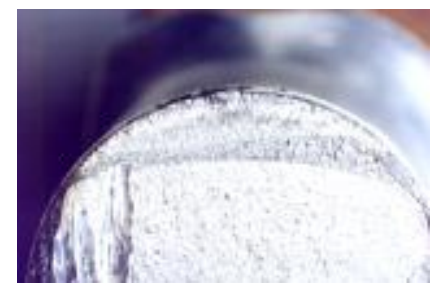

Р и с. 3. Сплав Д16Т: $\sigma_{a}=1,5 \cdot \sigma_{-1 \partial}$

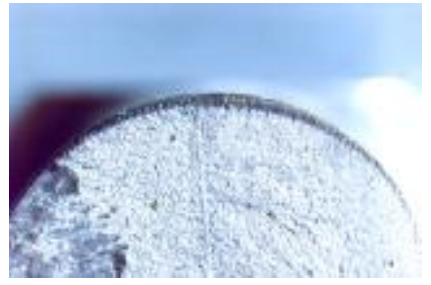

Р и с. 2. Сплав ЭИ961Ш: $\sigma_{a}=1,5 \cdot \sigma_{-1 \partial}$

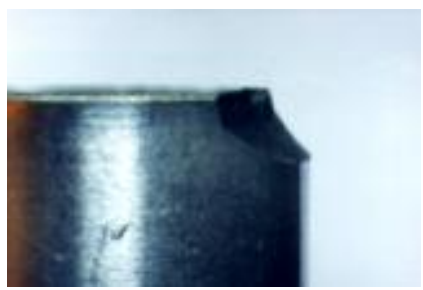

Р и с. 4. Сплав Д16Т : $\sigma_{a}=1,5 \cdot \sigma_{-1 \partial}$

Ответить на этот вопрос можно, если руководствоваться авторской методикой физикоматематического макроанализа как усталостного излома, так и статического излома деталей с так называемой нераспространяющейся усталостной трещиной $[3,4]$.

На рис. $5 a$, б,в изображены статические изломы образца, подвергшегося испытаниям на базе $N=10$ млн. циклов. На этих рисунках поверхность изломов представляет собой сложную бороздчатую форму (как результат сдвиговых деформаций). При макроувеличении (х4) зоны с нераспространяющейся усталостной трещиной (рис.5, верхнее прямоугольное выделение), которая, на первый взгляд, является плоской, отчетливо просматривается волновая поверхность (рис.5, б), период которой для стали 45 (наружный диаметр равен 25 мм, радиус полукруглого надреза - 1мм) составляет приблизительно 1 мм. На рис.5, в данное выделение в редакторе Fotoshop 6.0 преобразовано в режиме фильтрации по стилизации «выпуклый рисунок» (искаж.187, высота пиксел-76, кол.-439\%). Нижнее прямоугольное выделение на рис.5, а после 
преобразования в режиме фильтрации по стилизации «вывести контур» на уровне 210 с высоким краем изображено на рис.6. Здесь видна зона долома, соответствующая плоскому поперечному сечению. Фильтрация данной области позволила выявить плоские продольные сечения как результат микротрещин, не попадающих в глубину резко изображаемого пространства при фотографировании с макроувеличением.
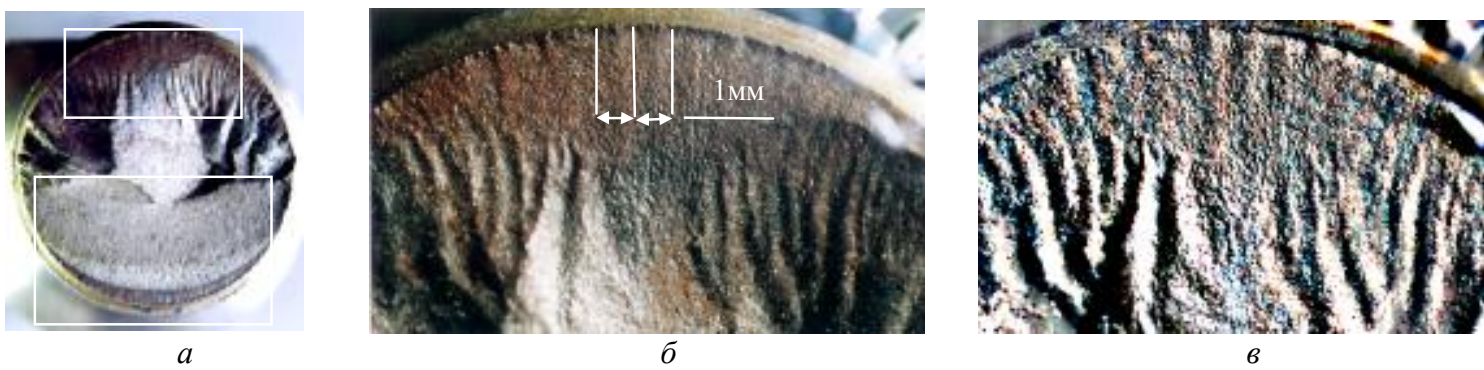

Р и с. 5. Статический излом образца из стали 45 после усталостных испытаний на изгиб при $\sigma_{a}=\sigma_{-1 \partial}$

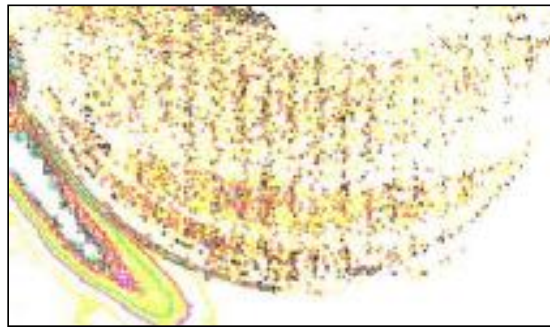

Р и с. 6

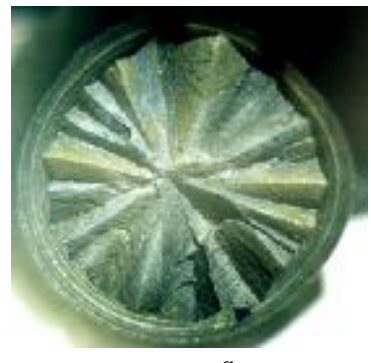

$a$

Если принять волновые поверхности как совокупность продольных сечений детали (или как плоскости поверхностного разрыва) [5], то, развивая математическую модель интегрирования дифференциальных поверхностей разрыва, можно создать теоретические модели и методы описания поверхностей перспективного разрушения в наиболее опасной зоне детали. Только в этом случае можно прогнозировать поверхность усталостного излома при кручении (рис.7, $а$, б, в), представляя деталь как совокупность радиально распределенных плоских продольных сечений.

Р и с. 7. Усталостные изломы образцов при кручении: $a$ - сталь $45, \mathrm{~V}$-образный надрез без упрочнения ППД; б-сталь $45, \mathrm{~V}$-обр. надрез, ППД- ГДО; в- Ст.45, полукруглый надрез $R=0,3 \mathrm{~mm}$, ГДО

Следовательно, возвращаясь к изгибу, деталь можно представить как совокупность плоских продольных сечений (рис.8), которые в опасной зоне при амплитуде напряжений от $\sigma_{-1 T p}$ до $\sigma_{-1 д}$ поворачиваются относительно центра изгиба 1 (рис. 9), представляющего собой нейтральную ось, определяя сдвиговые деформации в виде упругих волн.

Вероятность проявления действия гипотезы плоских продольных сечений зависит от концентрации напряжений в области изменения призматической формы детали. Так, при кручении цилиндрических образцов с кольцевым надрезом полукруглого профиля без наличия благоприятных остаточных напряжений в опасной зоне излом сначала имеет радиально сдвиговую форму фактически под действием волн сдвига, а затем, после снижения энергии радиальных сдвиговых деформации в поперечном сечении, переходит в разрушение сдвигом под углом $45^{\circ} \mathrm{\kappa}$ продольной оси (рис.10).

При центральном растяжении-сжатии энергия, направленная на распределение деформаций вдоль продольных сечений, проявляется слабо. На рис. 11 изображен усталостный излом цилиндрического образца из стали 45 при центральном растяжении-сжатии. В центральной части на рис. 11 видна направленная картина продвижения магистральной усталостной трещины, которая затем распределилась в плоскости поперечного сечения. 


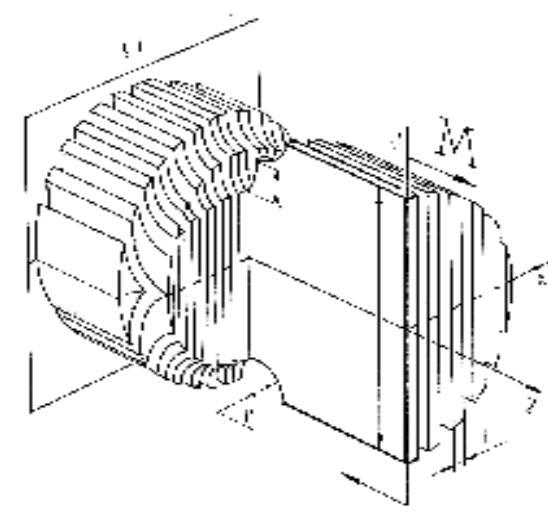

Р и с.8. Представление детали с усталостной трещиной при изгибе

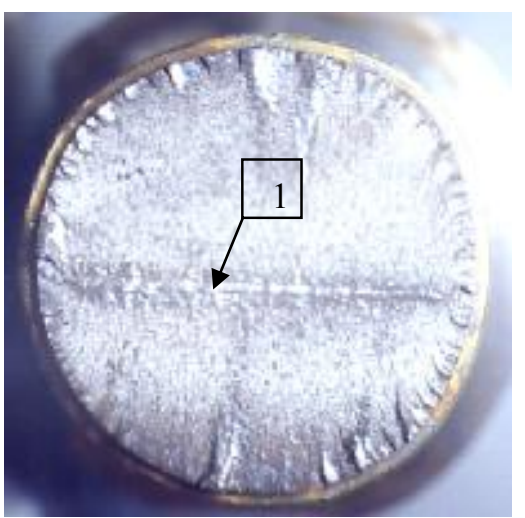

Р и с. 9. Сталь 45, изгиб, $\sigma_{a}=2,0 \cdot \sigma_{-l \partial}$

Необходимо отметить, что при сложном нагружении, в частности при кручении с изгибом, картина усталостного излома зависит от сочетания составляющих значений нормальных напряжений изгиба и касательных напряжений кручения На рис. 12 изображен усталостный излом при отношении $\sigma_{a} / \tau_{a}=1,2$ где при переходе нормального излома от изгиба к излому от сдвига при кручении видна волновая поверхность 1.

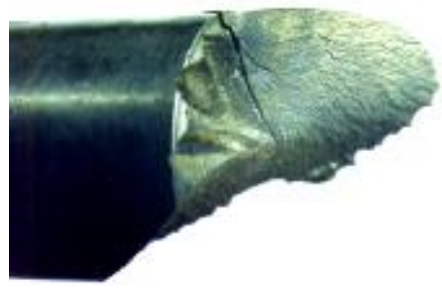

Р и с. 10. Излом при кручении

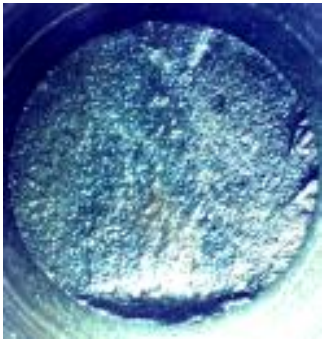

Р и с. 11.Излом при центральном растяжении-сжатии

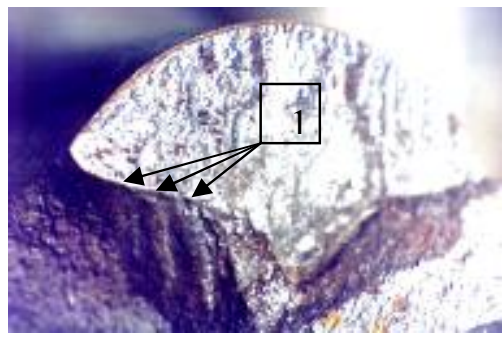

Р и с.12. Излом при кручении с изгибом

Таким образом, имея доказательную базу существования упругих продольных волн и волн сдвига не только при мгновенном нагружении (ударе) [ 5 ], но и при многоцикловом нагружении деталей, появляется возможность создания нового направления исследований в области механики разрушения, основанного на продольном распределении энергии внутренней деформации при различных видах простого и сложного нагружений деталей. Это позволяет ввести в механику сплошной среды новую гипотезу - гипотезу плоских продольных сечений.

Гипотеза автора настоящей статьи о плоских продольных сечениях, предназначенная для создания теоретических основ связи упругих волн при многоцикловом нагружении и потенциальной энергии внутренней деформации в механике сплошной среды, формулируется следующим образом: в условиях многоциклового нагружения деталей, в диапазоне амплитуд напряжений от предела выносливости по первой макротрещине до предела выносливости по разрушению, на характер формирования поверхности усталостного излома влияет распространение продольных и сдвиговых упругих волн, которые резонируя концентрируют потенциальную энергию внутренней деформации в плоских продольных сечениях, распределение которых в опасной зоне детали зависит от вида простого нагружения, в частности при изгибе плоские продольные сечения распределяются параллельно силовой плоскости с шагом, равным полупериоду упругой волны, при кручении - плоские продольные сечения распределяются радиально и зависят от конструктивного изменения призматической формы детали и остаточных напряжений.

Здесь необходимо отметить, что усталостные изломы при центральном растяжении-сжатии требуют более глубокого физико-математического микро- и макроанализа. Сложное сопротивление представляет собой в этом случае комбинацию параллельных и радиальных плоских продольных сечений. При этом форма излома представляет собой сложную искривленную поверхность. 
Руководствуясь гипотезой плоских продольных сечений, появляется возможность создания математических моделей или, точнее, расчетных методик прогнозирования поверхности излома при различных видах многоциклового нагружения деталей.

\section{БИБЛИОГРАФИЧЕСКИЙ СПИСОК}

1. Navier. Resume des lecons, donnees a l'Ecole des Ponts Chaussees sur l'applicaition de la mecanique a l'etablissement des constructhions et des machines. Premier partie contenante les lecons sur la resistance des materiaux. Paris. 1864.

2. Гуле Ж. Сопротивление материалов: Пер. с франц. А.С.Кравчука. М.: Высш.шк., 1985. 192с.

3. Кольцун Ю.И. Механика концентрации напряжений в окрестности фронта нераспространяющейся усталостной трещины // Вестн. Сам. гос. техн. ун-та. Сер.: Физ.-мат. науки. 2004. № 30. С.41-54.

4. Кольиун Ю.И. Локальный теоретический коэффициент концентрации напряжений при прогнозировании предела выносливости по первой макротрещине // Проблемы прочности материалов и сооружений на транспорте : Труды VI Международной научно-технической конференции / Под ред. С.В.Елизарова. СПб.: ПГУПС, 2004. C.181-190.

5. Ильюшин А.А., Ленский В.С. Сопротивление материалов. М.: Физматгиз, 1959. 371c.

Поступила 21.12.2004 2.

\section{Астрометрия и небесная механика}

УДК 521.1

Ф.Х. Алтынбаев

ЧИСЛЕННОЕ ИНТЕГРИРОВАНИЕ УРАВНЕНИЙ ДВИЖЕНИЯ НЕБЕСНЫХ ОБЪЕКТОВ МЕТОДОМ РАЗЛОЖЕНИЯ В РЯД ТЕЙЛОРА С УЧЕТОМ РЕЛЯТИВИСТСКИХ ЭФФЕКТОВ

Разработан алгоритм и составлена программа численного интегрирования уравнений движения небесных объектов методом Тейлора с учетом релятивистских членов, обусловленных Солнцем. На конечный момент времени для планеты Меркурий проведено сопоставление элементов орбит, полученных разными методами.

Раздел теоретической астрономии, изучающий движение небесных тел, можно считать основным разделом астрономических наук. Это изучение началось с открытия различного рода периодичностей в видимом движении небесных тел. После открытия закона всемирного тяготения и выявления его доминирующего значения основной задачей, ставшей перед астрономической дисциплиной, стал вывод всех следствий этого закона.

Основным содержанием небесной механики является задача $n$-тел, т.е. изучение движения $n$ материальных точек, притягивающихся друг к другу по закону Ньютона. Эта задача полностью решена аналитически только в случае $n=2$. При $n \geq 3$ точного аналитического решения не найдено. Задача трёх и более тел аналитическими методами решается путём разложения в степенные ряды по малым параметрам элементов орбит (эксцентриситет, наклонение). Однако это не позволяет моделировать движение с необходимой точностью на интервалах времени более ста лет.

В настоящее время при создании теориии движения небесных объектов широко используются численные методы интегрирования уравнений движения. Одним из высокоточных численных методов является метод, основанный на построении решения на следующем шаге, если оно известно на предыдущем, в виде ряда Тейлора. В современной численной теории движения небесных объектов, помимо гравитационных сил в уравнении учитываются релятивистские члены, которые, как известно, позволяют описать дополнительное смещение перигелия Меркурия, равное 43 с дуги за столетие.

В данной работе приведены формулы для учета релятивистких членов в методе численного интегрирования, основаном на представлении решения рядом Тейлора. По данному алгоритму проведено совместное интегрирование движения девяти больших планет (Меркурий-Плутон), и результаты интегрирования сопоставлены с результатами численного решения этой же задачи методом Эверхарта [1]. 